\title{
Role of Machine Learning \& Artificial Intelligence Techniques in Software Testing
}

\author{
Nilofar Mulla ${ }^{1}$, Dr. Naveenkumar Jayakumar ${ }^{2}$ \\ ${ }^{1}$ Bharati Vidyapeeth's College of Engineering for Women, Pune, Maharashtra, India \\ ${ }^{1}$ Bharati Vidyapeeth Deemed to be University College of Engineering, Pune, Maharashtra, India \\ ${ }^{2}$ Department of Computer Engineering, Bharati Vidyapeeth Deemed to be University College of \\ Engineering, Pune, Maharashtra, India \\ Email Ids:- nilofar.mulla2006@gmail.com \\ naveenkumar@bvucoep.edu.in
}

Article History: Received: 10 November 2020; Revised 12 January 2021 Accepted: 27 January 2021; Published online: 5 April 2021

\begin{abstract}
This study provides information about the use of artificial intelligence (AI) and machine learning (ML) techniques in the field of software testing. The use of AI in software testing is still in its initial stages. Also the automation level is lesser compared to more evolved areas of work.AI and ML can be used to help reduce tediousness and automate tasks in software testing. Testing can be made more efficient and smarter with the help of AI. Researchers recognize potential of AI to bridge the gap between human and machine driven testing capabilities. There are still number of challenges to fully utilize AI and ML techniques in testing but it will definitely enhance the entire testing process and skills of testers and will contribute in business growth. Machine learning research is a subset of overall AI research. The lifecycle of software is increasingly shortening and becoming more complicated. There is a struggle in software development between the competing pressures of developing software and meeting deadlines. AI-powered automated testing makes conducting full test suites in a timely manner on every change. In this article a detailed overview about the various applications of AI in software testing have been demonstrated. Also the implementation of machine learning in software testing has been discussed in detail and use of different machine learning techniques has been explained as well.
\end{abstract}

Keywords: machine learning techniques, artificial intelligence.

\section{Introduction}

Automation Test practices provides a lot of benefits to the software development process. However, they fail in some situations, in that case, Artificial Intelligence copes with those challenges and finds solutions to those challenges. Advancement in AI and machine learning helps the developers to perform various tests at once. Using the AI can save the time of the developers as well as can reduce the chances of bugs in the software testing. In this article, the various AI techniques used in software techniques and also the application of AI in software testing are discussed. How effectively AI can cope with the problems of automated test practices can also be seen in this article. AI also helps in the generation of API tests which helps the developer to do the software testing easily by identifying the risky areas. 


\section{Application of AI in software testing}

AI provides automation testing which increases the efficiency of the testing team. The quality of the test cases can be improved through Artificial Intelligence (Tuncali et al. 2018). Automation testing is one of the important applications of AI. Automation Testing allows the tester to run a lot of test cases at the same time. Automation Testing detects a minor bug and protects the testers from running a large test. The test cases offered by Artificial intelligence are easy to control and can execute very fast. AI helps the developer to save their time and choose the most efficient option while developing the test cases as in a couple of seconds. AI detects the best approaches to the test cases. When the developer changes the code, AI offers a minimum number of tests which has a positive or negative impact.

AI saves the time of the developers as it reduces the data redundancy and manual involvement during the testing. Eggplant AI, Appvance and Test.ai are the AI tools that are used for automated testing. Eggplant AI helps in detecting the technical issues that arise in the tested software and provides smart solutions to the problem. Graph reports on all activities can also be provided by the Eggplant. Appvance is used for predicting the performance of cognitive generation and users' behaviour. The screen-by-screen analysis is provided to the testers by this tool (Guo et al. 2017).

Self-healing the execution of Selenium tests- One of the best-automated testing frameworks in $\mathrm{AI}$ is known as Selenium. Selenium tests are the most complex testing which is the slowest executing test and the progress of all the test cases gets lost in this kind of Testing. Azeem et al. (2019) stated that, all the broken tests are identified by artificial intelligence and the broken tests are fixed by AI. Artificial Intelligence fixes the technical errors that arise during the execution of the code. The issues of the selenium tests can be healed through artificial intelligence automatically and also improve the test case. Selenium's test failure can be defined as the Artificial Intelligence heuristics and then issues are fixed and test run without stopping. AI can offer smart recommendations to the testing when one solution will not work.

Automating API test generation- Automating API is one of the important applications of AI in the testing process. The quality of interaction between different programs that communicate with the various protocols, databases and servers can be measured through the API evaluation. The stability of the connection and the correct processing of the requests are measured through testing. Di Nucci et al. (2018) stated that, the users can use multiple test cases with the API testing automation for the API QA. API testing automation also allows the developers to evaluate the functionality of multiple third-party tools. Automation is a must in cases when a lot of APIs work together. All the potential risky areas of the connected applications can be analyzed with artificial intelligence and provide ease to create test cases. The large data can be easily designed through the AI and can easily check the working of the API.

Visual validation automation Testing- Artificial Intelligence provides quality assurance to the developers during software testing. The displaying of the UI to the end-user is measured through quality assurance. Quality Assurance consists of visual validation testing. According to Khatibsyarbini et al. (2018), this testing is done to measure the correctness of UI that the UI elements appear to the end-users in the correct size, shape, position and colour. Testers face 
many difficulties in automating visual testing due to a large number of bugs. For the manual tester, it becomes a hectic task to identify and fix the bugs. In this case, AI helps in identifying the environment where the applications run, browsers, operating systems and determining the UI standards. Artificial Intelligence can verify the AI whether is appearing correctly to users.

\section{Implementation of machine learning in software testing}

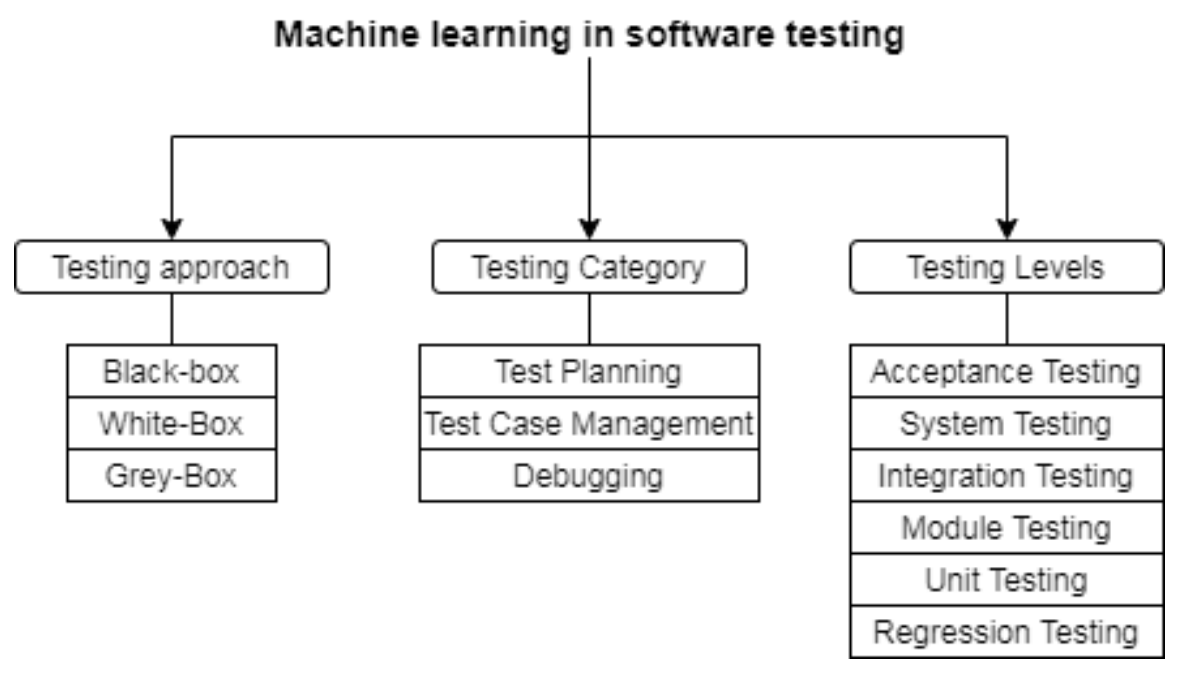

Figure 1: Machine learning in software testing as per current practises

(Sendra et al. 2017, p. 672)

Machine learning technique is an effective tool that can increase the effect of software testing processes. This technology can provide better understanding towards customers. Implementation of machine learning techniques in software testing can provide many opportunities for customers. Invariant testing approaches are maintained properly with the involvement of machine learning techniques, which directly has an impact on software testing. Machine learning technology is a common model of software testing. The software testing process is a fairly straightforward activity (Sendra et al. 2017). Along with this, disagreement and ambiguity of software testing processes are maintained with the involvement of machine learning technology. Predictive analytical approaches are also used in the maintenance of software testing. With the help of a few resources, it is noticed that machine learning technology is effective than predictive analytics. An effective layered algorithm is implemented successfully to maintain the efficiency of machine learning technology.

A high-performing algebraic coefficient is involved in the maintenance of machine learning technology. The effective electronic wind sensor is implemented successfully to maintain this neural network. Five separate equations are present in machine learning technology, which directly has an impact on the software testing process. Individual nodes are involved in the justification of machine learning technology. The implementation of machine learning technology in software testing is evaluated with the help of these technologies. Parallel computing technologies are present in machine learning technology (Wang and Lu 2019). Wind speed and direction are involved in the maintenance of 500 data points of the machine learning process, which directly has an impact on the implementation of this technology in software testing processes. This technology can play a crucial role in the maintenance of 
training data, which is crucial for software testing. Along with this quality of the network is evaluated with the help of machine learning technology.

The process of machine learning is used to analyze the functions on the level of the output. The function of software testing provides a scenario which is used in implementing the function of machine learning. The approach of software testing consists of different attributes based on the design of the process. The different activities are used in the practices of suitable aspects on the function of different analysis. Amershi et al. (2019) stated that, the access of data required a number of approaches to enable the operations of the system. There is an effective method are used on the basis of different aspects of information. The different attributes of the software required a resource which plays a significant role in the domain of the system. The concept of machine learning is implemented with the help of suitable strategies which are required to provide the method of testing in terms of a proper framework. The different capabilities of the software provide an effective analysis in order to validate the use of the given function.

The need of testing helps in the development of suitable models which are used to generate the accuracy of the system (Yang and Bang, 2019).The strategy of machine learning performs a function on the aspects of different methods which are required to describe the functions of the software. The fundamentals of machine learning are used to relate the concept of testing by regulating the methods of the given process. The dimensions of the testing create a test planning by managing the overall estimation of the system. The analysis of software generates the methods by reducing the development of the testing. The formation of the system collects suitable factors on the evaluation of the software which is used to increase the efficiency by maintaining the quality of the software. These are the processes which are used to implement the functions of machine learning in software testing (Melo et a. 2019).

\section{Overview of machine learning techniques}

Machine learning is referred to as one of the integral branches of artificial intelligence. If explained simply, it is a field of research that emphasizes teaching cognitive activities to machines. It helps artificially constructed machines to function as human minds. According to Arnold et al. (2019), contrary to the normal human mind and its capabilities, with the help of machine learning huge amounts of information can be stored within machine databases which can be programmed and developed according to specific necessity.

Machine learning consists of two primary categories, one of which is referred to as supervised learning while the other is named unsupervised learning. 

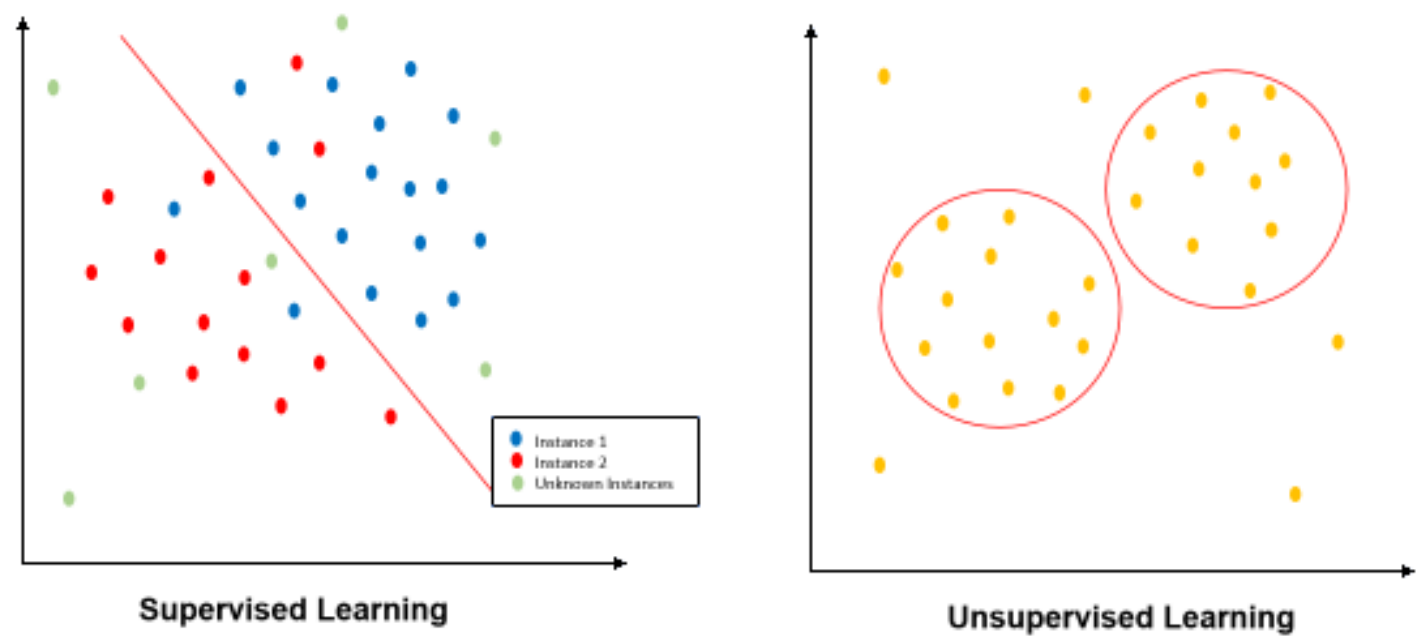

Figure 2: Supervised learning model vs unsupervised learning model

(Figure: Dwarakanath et al. 2018, p. 124)

Technically in supervised learning, there are two different variables into play. One of the variables is named (x) or the input variable, while the other is named $(\mathrm{y})$ or the output variable. The main goal of supervised learning is to formulate the mapping functions in such a way that after inputting a new value in the (x) the output data (y) can be predicted effectively. Conceptually, supervised machine learning translates to a set of input-out data pair which can be successfully mapped along the way of changing the input variable until and unless the output variable matches the requirement (Dwarakanath et al. 2018). The mathematical representation of supervised machine learning is $\mathrm{y}=\mathrm{f}(\mathrm{x})$. Supervised machine learning is further classified into classification machine learning techniques and regression machine learning techniques. Examples of classified techniques include discriminant analysis, Naive Boyes, nearest neighbour and support vector machines. Regression techniques include linear regression, decision trees, neural networks, SVR, GPR.

In the case of unsupervised machine learning, only the input variable value needs to be placed. The goal of unsupervised learning is to learn more about the input data by implementing it upon a database structure or a machine learning system. Conceptually, unsupervised learning is referred to such data algorithms which are untagged to each other. Contrary to the supervised machine learning process, unsupervised machine learning does not require human supervision and can work on itself. Maxwell et al. (2018) stated that, unsupervised machine learning is also further classified into 2 different groups, one of them known as clustering while the other named as an association. Clustering translates to such a technique that helps in the identification of inherent groupings within the selected data. Association machine learning techniques translate to the learning of the rules included in the data portions. It is also referred to as rulebased machine learning due to the fixed relations within its database. K-Means, K-Medoids, Hierarchical, Neural Networks, Hidden Markov Model. Apriori algorithm is mostly used for association based learning algorithms and problems. 
Apart from both the above-discussed machine learning forms, another secondary machine learning form is also present, which goes by the name of semi-supervised machine learning. These machine learning algorithms consist of a large amount of input data and a small amount of output data. It is also referred to as a mixture of supervised and unsupervised machine learning technique (Zhang et al. 2020).

\section{Evaluation of software testing techniques}

In the entire lifecycle of software, testing is required and done at each level of the development phases. These testing spectrums usually differ according to the nature and objective of the testing.

A basic graph of the popular testing process used in the industry now-a-days has been provided below:

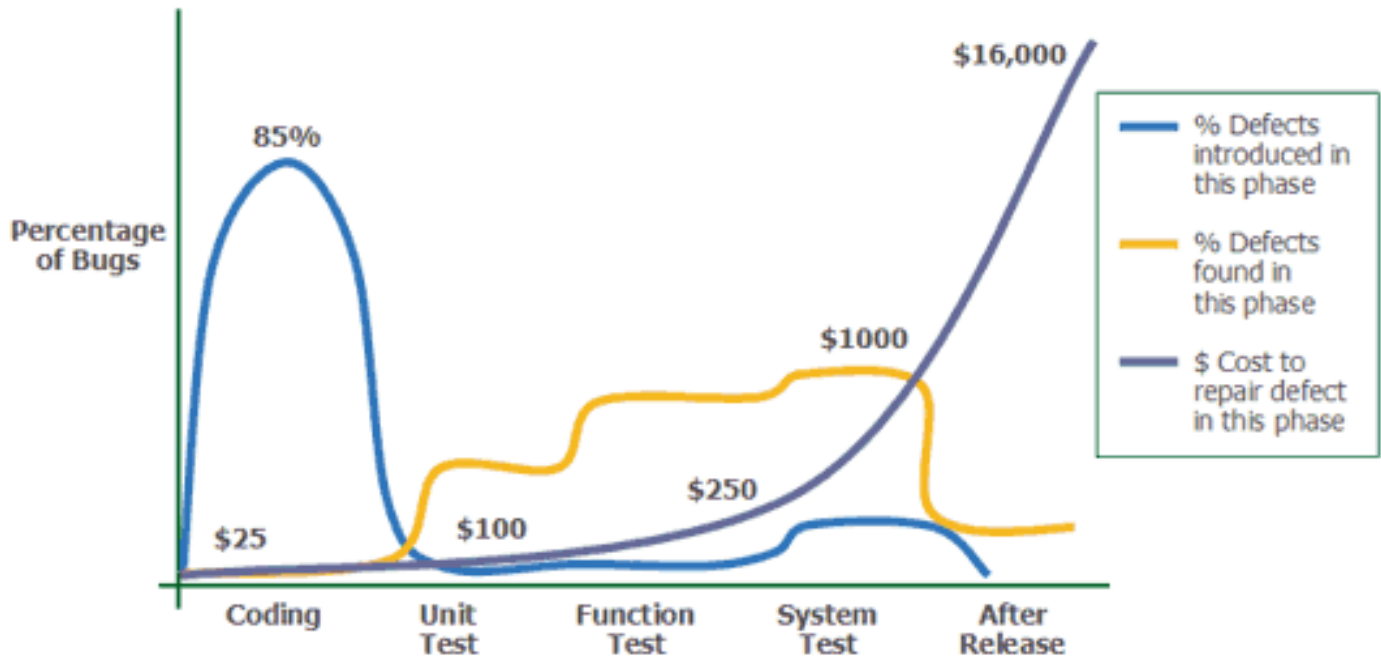

Figure 3: Basic testing spectrum in practise

(Figure: Musumeci et al. 2018, p.1387)

The first and foremost spectrum is referred to as unit testing. This testing is done in the preliminary stages of software development. The basic unit of the software is tested during this testing phase. The unit is also referred to as "module" and "component" depending upon the type of the software (Musumeci et al. 2018).

The next form of testing is referred to as integration testing. This technique or spectrum is performed when a larger structure is created from two or smaller tested units. The testing involves the testing of the larger structure as well as the smaller structures so that the quality of the software can be assessed and maintained.

The next testing spectrum is referred to as system testing. In this testing process, the quality of software from the beginning to its end is tested. It is also named such because of its testing procedure which involves the testing on an entire system (Seretis and Sarris 2021).

This is the last stage of the testing spectrum. This testing phase is referred to as acceptance testing as it involves the testing of the entire software system when the software is handed over 
to the users and customers. Through this process, the real-time usability of the software is assessed and it helps in increasing and establishing the confidence of the customers.

The above-discussed points showcase the different levels or stages of software testing. Conventionally, there are two main techniques for testing software. These are respectively known as manual and automated software testing techniques.

Manual testing translates to the testing of software with the help of automated testing tools. In this specific process of software testing, the respective tester creates different testing cases and codes. After that, he/she executes the tests and then reports the execution results to the developers and the respective project managers. The only demerit to this testing process is its resource and time consumption. Seo et al. (2020) stated that, it also requires the manual verification of the test results from the software tester in every phase of the testing procedure.

Automated software testing procedure, as the name suggests is a process of software testing that involves the use of automated tools to perform the testing process. It is a very efficient testing process and negates all the limitations that are present in the manual testing procedures.

Most software testers still prefer manual testing procedure simply because of the elevated confidence that the process establishes. But such a testing procedure is not possible for big sized software projects, as it will retain a huge amount of time from the entire software development process.

After the testing process is completed, an after-testing phase comes into play. This is also referred to as the test summary (Merghadi et al. 2020). It is usually a collection of all the logs and reports that were recorded during the entire testing procedure from beginning to end. It finalizes the launching of the software.

\section{Conclusion}

Artificial Intelligence plays an important role in the field of software testing. Machine learning is a subset of Artificial Intelligence and helps in realizing AI. AI can boost automation in testing, test case generation and can help in improving overall testing lifecycle. Visual validation automation testing in AI helps to check the UI standards for the various applications and check the correctness of the test done by the developers. Machine learning focuses on the decision-making capacity of the AI. The software now a day is becoming more and more complex. The use of AI will definitely change testing and test automation as well. AI can also be widely used for mobile applications testing. Machine learning algorithms help learning from test results from same data which can help in training decision making AI. 


\section{References}

[1]. Amershi, S., Begel, A., Bird, C., DeLine, R., Gall, H., Kamar, E., Nagappan, N., Nushi, B. and Zimmermann, T., 2019, May. Software engineering for machine learning: A case study. In 2019 IEEE/ACM 41st International Conference on Software Engineering: Software Engineering in Practice (ICSE-SEIP) (pp. 291-300). IEEE.

[2]. Arnold, M., Bellamy, R.K., Hind, M., Houde, S., Mehta, S., Mojsilović, A., Nair, R., Ramamurthy, K.N., Olteanu, A., Piorkowski, D. and Reimer, D., 2019. FactSheets: Increasing trust in AI services through supplier's declarations of conformity. IBM Journal of Research and Development, 63(4/5), pp.6-1.

[3]. Azeem, M.I., Palomba, F., Shi, L. and Wang, Q., 2019. Machine learning techniques for code smell detection: A systematic literature review and meta-analysis. Information and Software Technology, 108, pp.115-138.

[4]. Di Nucci, D., Palomba, F., Tamburri, D.A., Serebrenik, A. and De Lucia, A., 2018, March. Detecting code smells using machine learning techniques: are we there yet?. In 2018 ieee 25th international conference on software analysis, evolution and reengineering (saner) (pp. 612621). IEEE.

[5]. Dwarakanath, A., Ahuja, M., Sikand, S., Rao, R.M., Bose, R.J.C., Dubash, N. and Podder, S., 2018, July. Identifying implementation bugs in machine learning based image classifiers using metamorphic testing. In Proceedings of the 27th ACM SIGSOFT International Symposium on Software Testing and Analysis (pp. 118-128).

[6]. Guo, J., Cheng, J. and Cleland-Huang, J., 2017, May. Semantically enhanced software traceability using deep learning techniques. In 2017 IEEE/ACM 39th International Conference on Software Engineering (ICSE) (pp. 3-14). IEEE.

[7]. Khatibsyarbini, M., Isa, M.A., Jawawi, D.N. and Tumeng, R., 2018. Test case prioritization approaches in regression testing: A systematic literature review. Information and Software Technology, 93, pp.74-93.

[8]. Maxwell, A.E., Warner, T.A. and Fang, F., 2018. Implementation of machine-learning classification in remote sensing: An applied review. International Journal of Remote Sensing, 39(9), pp.2784-2817.

[9]. Melo, S.M., Moura, F.M., Souza, P.S. and Souza, S.R., 2019, September. Selectt: An infrastructure for selection of concurrent software testing techniques. In Proceedings of the IV Brazilian Symposium on Systematic and Automated Software Testing (pp. 62-71).

[10]. Merghadi, A., Yunus, A.P., Dou, J., Whiteley, J., ThaiPham, B., Bui, D.T., Avtar, R. and Abderrahmane, B., 2020. Machine learning methods for landslide susceptibility studies: A comparative overview of algorithm performance. Earth-Science Reviews, p.103225.

[11]. Musumeci, F., Rottondi, C., Nag, A., Macaluso, I., Zibar, D., Ruffini, M. and Tornatore, M., 2018. An overview on application of machine learning techniques in optical networks. IEEE Communications Surveys \& Tutorials, 21(2), pp.1383-1408.

[12]. Sendra, S., Rego, A., Lloret, J., Jimenez, J.M. and Romero, O., 2017, May. Including artificial intelligence in a routing protocol using software defined networks. In 2017 IEEE International Conference on Communications Workshops (ICC Workshops) (pp. 670-674). IEEE.

[13]. Seo, H., Badiei Khuzani, M., Vasudevan, V., Huang, C., Ren, H., Xiao, R., Jia, X. and Xing, L., 2020. Machine learning techniques for biomedical image segmentation: An overview of technical aspects and introduction to state-of-art applications. Medical physics, 47(5), pp.e148e167.

[14]. Seretis, A. and Sarris, C.D., 2021. An Overview of Machine Learning Techniques for Radiowave Propagation Modeling. arXiv preprint arXiv:2101.11760. 
[15]. Tuncali, C.E., Fainekos, G., Ito, H. and Kapinski, J., 2018, June. Simulation-based adversarial test generation for autonomous vehicles with machine learning components. In 2018 IEEE Intelligent Vehicles Symposium (IV) (pp. 1555-1562). IEEE.

[16]. Wang, Q. and Lu, P., 2019. Research on application of artificial intelligence in computer network technology. International Journal of Pattern Recognition and Artificial Intelligence, 33(05), p.1959015.

[17]. Yang, Y.J. and Bang, C.S., 2019. Application of artificial intelligence in gastroenterology. World journal of gastroenterology, 25(14), p.1666.

[18]. Zhang, J.M., Harman, M., Ma, L. and Liu, Y., 2020. Machine learning testing: Survey, landscapes and horizons. IEEE Transactions on Software Engineering. 